\title{
MENOS NUTRIENTES E MAIS ALIMENTOS: PROPOSTA DE ABORDAGEM PARA ESCOLHAS CONSCIENTES
}

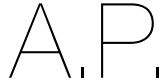

ARTIGOPROFSSIONAL

${ }^{1}$ Escola Superior de Tecnologia da Saúde de Lisboa,

Av. D. João II, Lote

4.69 .01

Parque das Nações,

1990-096 Lisboa, Portugal

2 CITUR - Centro

de Investigação,

Desenvolvimento e

Inovação em Turismo

Pólo do Estoril,

Avenida Condes de

Barcelona, n. ${ }^{\circ} 808$

2769-510 Estoril, Portugal

${ }^{3}$ GreenUPorto - Centro de Investigação em Produção Agroalimentar Sustentável Edifício de Ciências Agrárias (FCV2)

Rua da Agrária, n. ${ }^{\circ} 747$

4485-646 Vairão, Portugal

4 LAQV-Requimte da Universidade do Porto, 4051-401 Porto, Portugal

${ }^{5}$ Faculdade de Ciências da Nutrição e Alimentação da Universidade do Porto, Rua do Campo Alegre, n. ${ }^{\circ} 823$,

4150-177 Porto, Portugal

‘Endereço para correspondência:

Ada Rocha

Faculdade de Ciências da Nutrição e Alimentação da Universidade do Porto, Rua do Campo Alegre, n. 823, 4150-177 Porto, Portugal ada.margarida.rocha@gmail.com

Histórico do artigo:

Recebido a 1 de novembro de 202 Aceite 29 de dezembro de 2020

\section{CHOOSING FOOD OVER NUTRIENTS: PROPOSAL OF AN APPROACH FOR MORE CONSCIOUS CHOICES}

\section{RESUMO}

As preocupações com a alimentação e nutrição fazem parte da realidade dos dias de hoje, existindo uma cada vez maior atenção por parte do público em relação às características nutricionais dos alimentos que escolhem e consomem. A abordagem focada nos nutrientes é reducionista e dificulta a compreensão do consumidor sobre a melhor forma de avaliar a sua ingestão alimentar. No entanto, esta continua a ser a prática que prevalece em todos os contextos, quer na indústria alimentar, na restauração, na publicidade e nos supermercados. O objetivo deste trabalho foi desenvolver uma abordagem disruptiva de aplicação das recomendações alimentares veiculadas pela Roda dos Alimentos. Com base neste guia foi desenvolvida uma infografia a partir dos valores de referência médios para os adultos. A disponibilização desta informação nas ementas, menus, receitas, entre outros, assim como nos produtos alimentares permitiria facilitar a consciencialização da quantidade consumida em relação às porções diárias, assim como melhorar a perceção do que é uma porção para os vários tipos de alimentos, e de como o conjunto das diferentes refeições e produtos alimentares permitem atingir as recomendações alimentares e nutricionais.

\section{PALAVRAS-CHAVE}

Porções alimentares, Recomendações alimentares, Roda dos alimentos

\section{ABSTRACT}

Consumer's awareness about food and nutrition have increased in the last years, drawing attention to the nutritional properties of foods, namely their content in sugar, fat, calories and salt. The nutrient-focused approach is reductionist, and moreover, it is difficult for consumers to understand the best way to assess their food intake. However, in all contexts, this remains the methodology that prevails (food industry, restaurants, advertising, and supermarkets). The objective of this work was to develop a disruptive approach to the application of food recommendations conveyed by the Portuguese Food Guide. Using this guide, an infographic was developed based on the average reference values for adults. The availability of this information in menus, recipes, among others, as well as in food products would allow the awareness of the amount of food consumed in relation to the daily portions, as well as improving the perception of what is a portion, considering the various types of food and how different meals contribute to the daily recommendations.

KEYWORDS

Food portions, Food recommendations, Food guide

\section{INTRODUÇÃO}

A esperança média de vida dos portugueses tem vindo a aumentar nas últimas décadas, bem como a mortalidade prematura por causas relacionadas com os estilos de vida, no qual se inclui a alimentação (1). A relação entre a alimentação e a saúde é amplamente reconhecida pela comunidade científica e pelo público em geral (2). O Padrão Alimentar Mediterrânico é descrito como um modelo de alimentação saudável e sustentável a adotar, pelas implicações positivas em termos de saúde, estando claramente associado à redução do risco de doenças crónicas (doenças cardiovasculares, diabetes, alguns tipos de cancro, obesidade), à gestão do peso, e ao aumento da longevidade, mas também pela consciência ambiental, de proximidade à terra e à tradição que o mesmo propõe (2-7). Por outro lado, o consumo de alimentos processados, produtos açucarados, ricos em sal, gordura, gordura saturada e energia, característicos das sociedades de consumo atuais, associa-se ao desenvolvimento de doenças crónicas. A prevalência destas doenças continua a aumentar, apesar de todo o conhecimento que se tem vindo a construir $(2,8,9)$. As preocupações com alimentação e nutrição fazem parte da realidade dos dias de hoje, existindo uma cada vez maior atenção por parte do público em relação às características nutricionais dos alimentos que escolhem e consomem. Num estudo da Nielsen de 2016, os portugueses demonstraram, uma preocupação com a saúde superior à da média europeia na escolha de produtos alimentares (10).

Atualmente, existe uma grande variedade de produtos 
alimentares disponíveis, que procuram responder às atuais preocupações dos consumidores, não apenas em termos de saúde, mas cada vez mais, também, de sustentabilidade. A abordagem alimentar do consumidor está focada em mais proteína, menos sal, menos gordura, menos açúcar, menor teor de gordura saturada, menos colesterol, mais fibra, mais vitaminas e antioxidantes. A resposta da indústria alimentar traduz-se em produtos que evidenciam estas características específicas (11).

São exemplo disso iogurtes com teor elevado de proteína, zero açúcar adicionado, $0 \%$ de gordura, batatas fritas com redução de gordura, cereais de pequeno almoço com maior teor de fibra, sumos com maior teor de vitaminas e antioxidantes, fiambre sem sal, entre outros.

No entanto a evidência científica recente demonstra que são os padrões alimentares e não a ingestão de nutrientes específicos isoladamente que influenciam a saúde $(12,13)$.

A abordagem focada nos nutrientes é reducionista e dificulta a compreensão do consumidor sobre a melhor forma de avaliar a sua ingestão alimentar $(14,15)$. No entanto, esta continua a ser a prática que prevalece em todos os contextos, quer na indústria alimentar, na restauração, na publicidade, e nos locais de venda.

Este facto está patente nas práticas que evidenciamos: é comum vermos o valor nutricional associado às receitas, às ementas escolares, às ementas da restauração comercial, entre outras.

Tomando como exemplo a Figura 1, a informação apresentada evidencia que o produto em causa fornece $52 \%$ das necessidades diárias de proteína, $16 \%$ de hidratos de carbono, 36\% de lípidos, 37\% de sal e $25 \%$ da energia, dando foco aos nutrientes e à energia consumida, ao mesmo tempo que torna difícil a identificação de quais os alimentos a consumir nas restantes refeições para cumprir as recomendações. Ao responder a $52 \%$ das necessidades em proteínas fica claro que faltam $48 \%$, no entanto fica por saber a quantas gramas de proteína correspondem esta percentagem e a que quantidade de alimentos se refere. Este é apenas um exemplo, cuja interpretação é válida e transversal para qualquer alimento em que seja apenas e só, apresentada a quantidade e/ou contribuição percentual dos nutrientes em relação às necessidades diárias.

Acresce que o foco nos nutrientes desvaloriza o tipo de alimentos envolvidos, que no exemplo em concreto é bastante significativo. Não importa apenas saber a quantidade de hidratos de carbono, mas o tipo, uma vez que este fator condiciona o seu metabolismo, absorção e efeitos no organismo (16-18).

Por outro lado, o foco nos nutrientes leva a uma confusão muito frequente entre alimentos e nutrientes, comum entre os consumidores e entre os operadores do setor que disponibilizam esta informação ao público.

Referenciar "proteína" como ingrediente de prato é uma prática frequente, da qual são exemplo as imagens que apresentamos - Figura 2.

Para acrescer a esta confusão, a informação nutricional não é clara e objetiva nos rótulos dos produtos alimentares, persistindo a análise

\section{Figura 1}

Valor nutricional do produto ${ }^{1}$

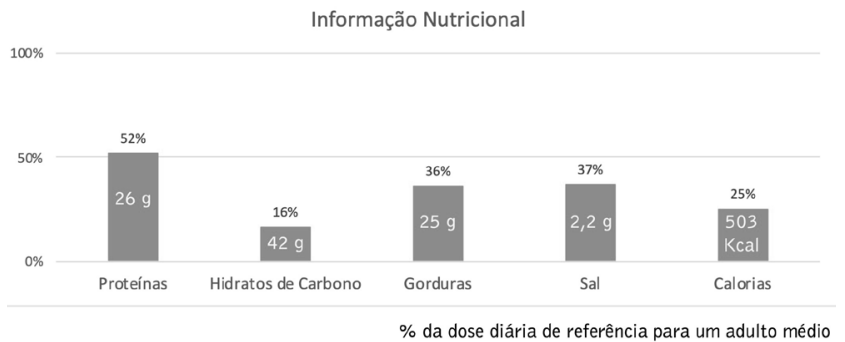

1 Informação referente a um hambúrguer disponível numa cadeia de fast-food em Portugal.
Figura 2

Exemplos ilustrativos de referenciação de nutrientes como alimentos crie o seu bowl

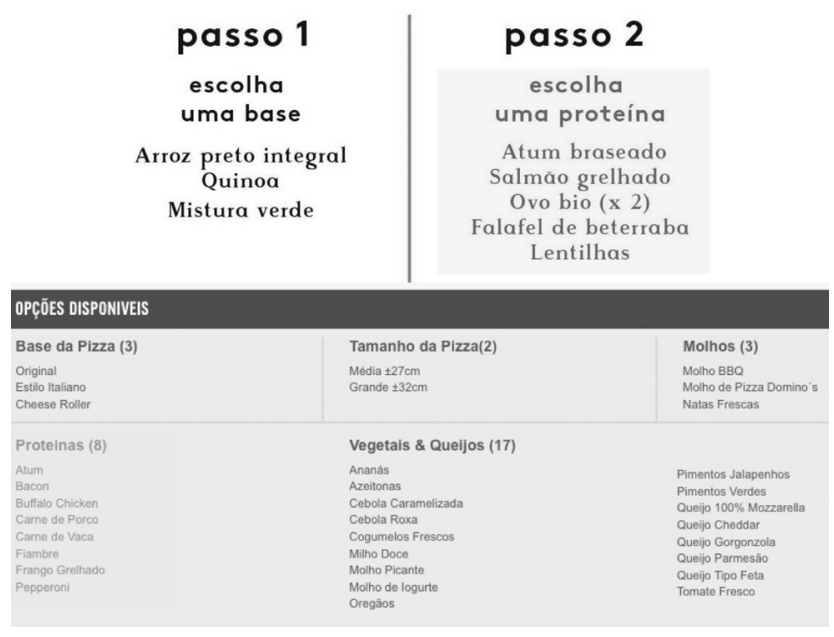

individual por nutriente e/ou valor energético, assim como a partilhada em receitas, livros de cozinha e saúde, ou revistas de diversos âmbitos e ainda em ementas (19).

Desde 1977 que Portugal dispõe de um instrumento de educação alimentar simples, fácil de utilizar e entender, que agrupa os alimentos em função do seu valor nutricional - roda dos alimentos. Este instrumento revisto em 2003 indica-nos o número de porções de cada um dos grupos alimentares, a ingerir diariamente (20). Apesar da generalidade dos indivíduos conhecerem os instrumentos de educação alimentar, a relação prática das porções com os pesos e quantidades dos alimentos é desconhecida $(21,22)$. Acresce que este instrumento de educação alimentar reflete o Padrão Alimentar Mediterrânico reconhecido como saudável e sustentável. Inclusivamente, foi recentemente atualizado, com o objetivo de introduzir e/ou reforçar alguns conceitos importantes no contexto da alimentação mediterrânica, tais como a importância dos frutos oleaginosos, a sazonalidade, o consumo de cereais integrais, entre outros (23). No entanto, não existe nenhuma ferramenta user friendly para aferir o grau de conformidade com este guia.

Neste sentido os autores propõem uma abordagem disruptiva de aplicação das recomendações alimentares veiculadas pela Roda dos Alimentos, de forma a permitir uma melhor compreensão daquilo que deve ser a alimentação, transferindo o foco das recomendações nutricionais para as recomendações alimentares.

\section{OBJETIVOS}

Desenvolvimento de infografia representativa das recomendações das porções alimentares da Roda dos Alimentos e sua potencial aplicação em diferentes contextos das áreas de atuação do nutricionista.

\section{METODOLOGIA}

Tomando por base as porções da roda dos alimentos (20) e os seus intervalos, os autores propõem a criação de um valor de referência médio para os adultos, a exemplo do que é feito com as recomendações nutricionais (2000 Kcal).

Desta forma foram consideradas para um adulto médio as seguintes recomendações alimentares apresentadas na Tabela 1, assumindo-se sempre valores inteiros para as porções médias para um adulto.

A satisfação das necessidades nutricionais - Tabela 2 - pode ser assegurada através de combinações diferentes de alimentos, tendo por base estes intervalos de porções para os diferentes grupos alimentares. 
Tabela 1

Recomendações alimentares (porções) para um adulto médio

\begin{tabular}{lcc}
$\begin{array}{c}\text { GRUPO DE ALIMENTOS DA RODA } \\
\text { DOS ALIMENTOS }\end{array}$ & $\begin{array}{c}\text { INTERVALO } \\
\text { DE PORCOES } \\
\text { RECOMENDADAS }\end{array}$ & $\begin{array}{c}\text { VALOR MÉDIO PARA } \\
\text { UM ADULTO }\end{array}$ \\
\hline Hortícolas & $3-5$ & $3-4$ \\
\hline Fruta & $3-5$ & $3-4$ \\
\hline Cereais, derivados e tubérculos & $4-11$ & $6-8$ \\
\hline Leguminosas & $1-2$ & $1-2$ \\
\hline Gorduras e óleos & $1-3$ & $2-3$ \\
\hline Laticínios & $2-3$ & $2-3$ \\
\hline Carne, pescado e ovos & $1,5-4,5$ & $3-4$ \\
\hline
\end{tabular}

Tabela 2

Recomendações nutricionais (macronutrientes) para um adulto médio (24)

\begin{tabular}{lcccccc} 
& \multicolumn{3}{c}{ VALOR ENERGÉTICO - DIÁRIO 2000 KCAL } \\
\hline & $\begin{array}{l}\text { HIDRATOS DE } \\
\text { CARBONO }\end{array}$ & \multicolumn{2}{c}{ LíPIDOS } & \multicolumn{2}{c}{ PROTEÍNA } \\
\hline $\begin{array}{l}\text { Distribuição } \\
\text { percentual (\%) }\end{array}$ & 50 & 60 & 20 & 30 & 10 & 15 \\
\hline Quantidade (g) & 250 & 300 & 45 & 67 & 50 & 75 \\
\hline
\end{tabular}

\section{RESULTADOS}

Com base nos valores médios referidos na Tabela 1, foi desenvolvida uma infografia representativa das recomendações das porções, considerando o valor máximo (Figura 3).

\section{Aplicabilidade em contexto de prática profissional}

Tomando como referência a infografia proposta, apresentam-se várias possibilidades de aplicação desta abordagem em diferentes contextos. O primeiro exemplo é referente a uma ficha técnica, igualmente aplicável a receitas em livros, websites ou programas de televisão, entre outros (Figura 4).

Mais do que a informação nutricional que consta na ficha técnica, a infografia com as porções, demonstra de forma mais clara a relação das quantidades de alimentos propostos para a refeição com as recomendações alimentares, evidenciando-se nesta refeição o fornecimento quase integral das porções relativas ao grupo da carne, pescado e ovos.

A associação da infografia a imagens das receitas e/ou pratos confecionados (Figura 5), assim como a representação desta informação na ementa para o consumidor (Figura 6), evidencia a relação das quantidades servidas/ingeridas com a satisfação das recomendações alimentares, permitindo ainda melhorar a perceção da correspondência de uma porção para os vários tipos de alimentos.

A utilização desta infografia em contexto de registo para planos alimentares (Figuras 7, 8 e 9), permitiria não apenas o anteriormente referido, como também a melhor compreensão de como as diferentes refeições e produtos alimentares podem ser conjugados de forma a possibilitar a satisfação das recomendações alimentares, assim como uma melhor perceção dos excessos e carências em relação a grupos ou alimentos. Por outro lado, pode ainda aumentar a consciencialização relativamente ao consumo de produtos alimentares que não se enquadram em nenhum dos grupos, como snacks salgados, doces e sobremesas, que devem constituir exceções e não a regra.

\section{ANÁLISE CRÍTICA}

A alimentação é muito mais do que a soma dos nutrientes contidos nos alimentos ingeridos. O prazer à mesa decorrente quer do convívio social, quer das sensações que os alimentos nos proporcionam é parte fundamental do ato de comer (25). Além destas questões,

\section{Figura 3}

Infografia representativa das porções alimentares a consumir diariamente par um adulto de referência²

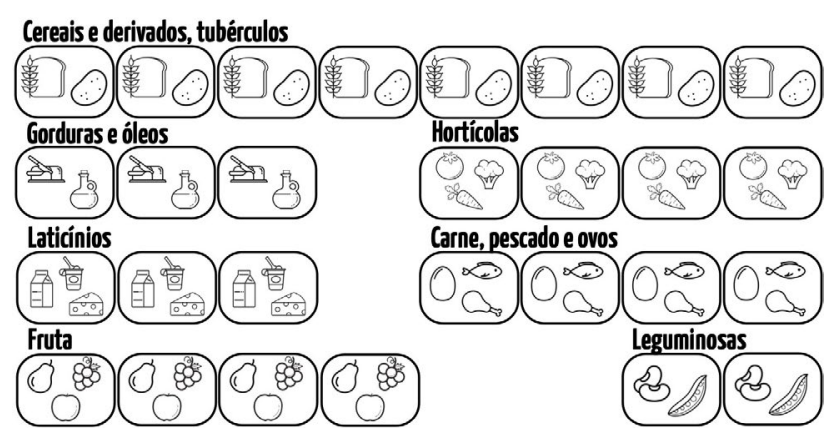

${ }^{2}$ Imagens retiradas de https://icons8.com e https://www.iconfinder.com

\section{Figura 4}

Aplicação da infografia em ficha técnica ${ }^{3}$

\begin{tabular}{|c|c|c|c|c|c|}
\hline \multicolumn{6}{|c|}{ Composiçáo nutricional de Arroz ao fomo } \\
\hline Ingrediente & Captaçāo & Prot & $\mathrm{HC}$ & Gord & VE \\
\hline Arroz & $50 \mathrm{~g}$ & $3,4 \mathrm{~g}$ & $39,1 \mathrm{~g}$ & $0,2 \mathrm{~g}$ & $172 \mathrm{kcal}$ \\
\hline Manteiga & $5 \mathrm{~g}$ & vest & vest. & $4,1 \mathrm{~g}$ & $37 \mathrm{kcal}$ \\
\hline Peito de peru cozido desfiado & $100 \mathrm{~g}$ & $23,4 \mathrm{~g}$ & $\mathbf{o g}$ & $1.3 \mathrm{~g}$ & $105 \mathrm{kcal}$ \\
\hline Azeitonas pretas descaroçadas & $6 \mathrm{~g}$ & $0,1 \mathrm{~g}$ & $\mathbf{o g}$ & $1,1 \mathrm{~g}$ & $10 \mathrm{kcal}$ \\
\hline Millo & $15 \mathrm{~g}$ & $1,4 \mathrm{~g}$ & $10,5 \mathrm{~g}$ & $0,7 \mathrm{~g}$ & $55 \mathrm{kcal}$ \\
\hline Queijo parmesáo ralado & $5 \mathrm{~g}$ & $1,9 \mathrm{~g}$ & og & $1,4 \mathrm{~g}$ & $20 \mathrm{kcal}$ \\
\hline Orégăos & $0,1 \mathrm{~g}$ & vest & vest. & og & - \\
\hline TOTAL & & $\begin{array}{l}30.2 \mathrm{~g} \\
30.3 \%\end{array}$ & $\begin{array}{r}49,6 \mathrm{~g} \\
4977 \%\end{array}$ & $\begin{array}{l}8,8 \mathrm{~g} \\
194 \%\end{array}$ & $399 \mathrm{kcal}$ \\
\hline
\end{tabular}

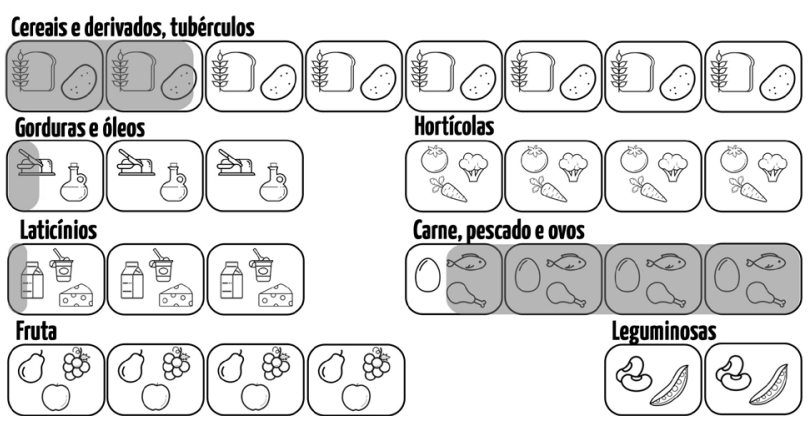

${ }^{3}$ A ficha técnica representada foi retirada do documento Orientações sobre Ementas e Refeitórios Escolares (https://www.dge.mec.pt/sites/default/files/Esaude/oere.pdf).

\section{Figura 5}

Aplicação da infografia em receitas ${ }^{4}$
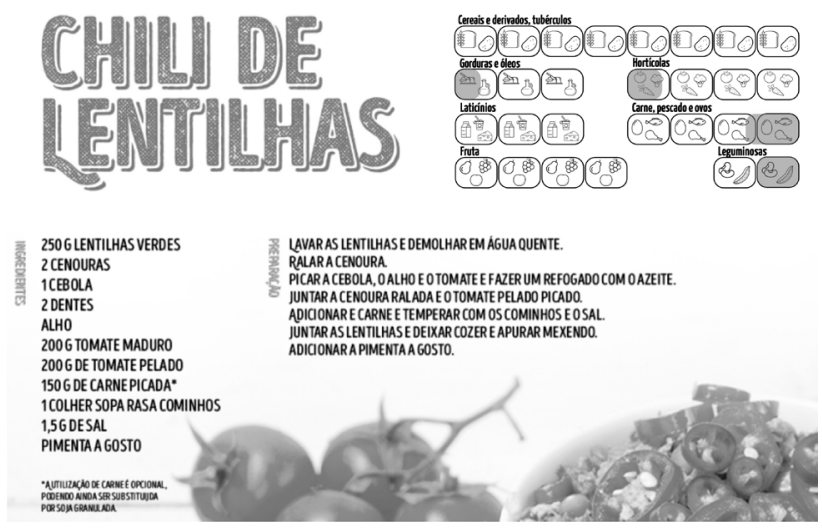

${ }^{4}$ Receita criada no âmbito do Ano internacional das leguminosas (2016), pela equipa de coordenação do Mestrado em Inovação em Artes Culinárias (ESHTE). 
o tipo, o volume, o aspeto, o aroma ou a textura daquilo que é consumido tem efeitos fisiológicos diferentes, condicionando a satisfação, a saciedade e a forma como os nutrientes são processados e absorvidos $(26,27)$.

Assim, a abordagem alimentar deve ser baseada no tipo de alimentos e não apenas condicionada pelo teor de nutrientes, até porque, a seleção adequada dos alimentos permite, por um lado a ingestão de maior quantidade de alimentos (ou uma maior margem em relação às quantidades consumidas) e, por outro, garante o aporte em nutrientes (salvo em situações e condições de saúde específicas).

A partir do valor médio de referência de $2000 \mathrm{Kcal}$, enquanto valor

\section{Figura 6}

Aplicação da infografia numa ementa

\begin{tabular}{|c|c|c|c|c|c|}
\hline \multirow{5}{*}{ 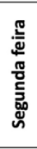 } & Refeição & $\begin{array}{l}\text { HC } \\
\text { (g) }\end{array}$ & $\begin{array}{l}\text { Lip } \\
\text { (g) }\end{array}$ & $\begin{array}{l}\text { Prot } \\
\text { (g) }\end{array}$ & $\begin{array}{l}\text { Energia } \\
\text { (Kcal) }\end{array}$ \\
\hline & Sopa de feijão verde & 10,8 & 3,0 & 1,8 & 78 \\
\hline & Salada de alface, tomate e cenoura, arroz ao forno com peru & 49,6 & 8,8 & 30,2 & 399 \\
\hline & Maçã & 19,3 & 0,7 & 0,3 & 84,8 \\
\hline & Total & 79,7 & 12,5 & 32,3 & 562 \\
\hline
\end{tabular}

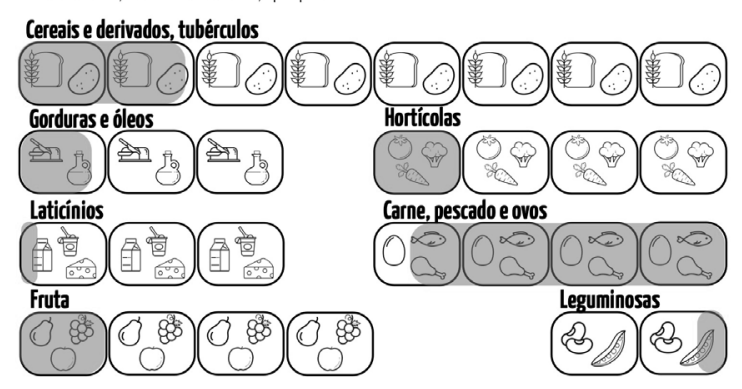

energético a atingir diariamente, foram considerados intervalos das porções dos diferentes grupos de alimentos, uma vez que as necessidades em nutrientes podem ser satisfeitas por combinações diferentes dos vários grupos, garantindo uma maior diversidade na ingestão alimentar, quer em termos qualitativos, quer quantitativamente. Por uma questão de simplicidade visual, a infografia apresenta sempre o valor máximo de porções. No entanto, a adoção desta abordagem, pode ser operacionalizada, considerando diferentes representações, de acordo com o grupo etário a que se destina.

A abordagem alimentar permite ainda olhar para o alimento de forma integral, possibilitando uma maior ligação com os alimentos, potenciando a reflexão sobre a sua proveniência, o seu cultivo ou o recurso a produções de proximidade, reduzindo o impacto dos transportes no meio ambiente e contribuindo para a solidez económico-financeira dos mercados nacionais/locais $(11,13)$.

A complexidade da informação atualmente disponível, e a falta de harmonização na forma como esta é apresentada entre os diferentes produtos/marcas, são referidas pelos consumidores como dificuldades à compreensão da mesma. O baixo índice de literacia da população portuguesa é apresentado por diversos autores como uma dificuldade adicional (28). Acresce que esta informação é centrada nos nutrientes, sendo cada vez mais frequentes, os exemplos de confusão entre nutrientes e alimentos, a sua utilização errónea como sinónimos e ainda os primeiros apresentados em substituição dos segundos. Esta confusão, afasta-nos da ligação natural que devemos ter com os alimentos, a sua origem, modo de produção, formas de confeção, entre outros aspetos que têm impacto nas questões da sustentabilidade, além das ligadas às tradições e à cultura alimentar.

\section{Figura 7}

Exemplo de um registo diário

As porções que deve consumir diariamente são* ...

Cereais, derivados e tubérculos - 6 a 8 porç̃es

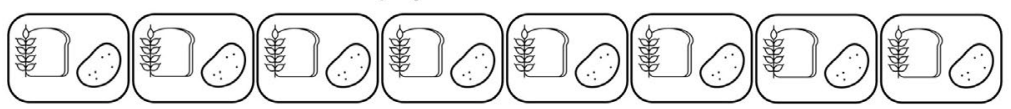

Horticolas - 3 a 4 porções

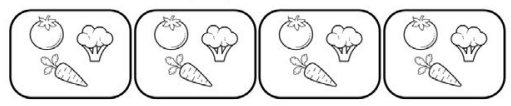

Carne, pescado e ovos - 3 a 4 porções

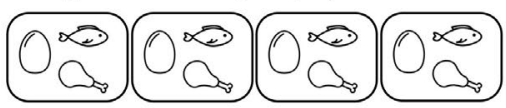

Fruta - 3 a 4 porções

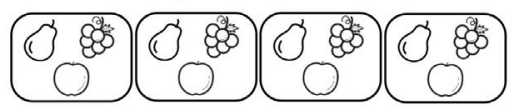

Laticínios - 2 a 3 porções

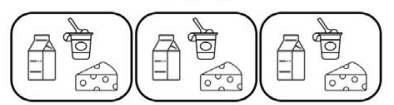

Leguminosas - 1 a 2 porções

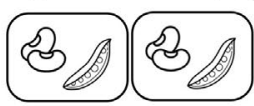

Gorduras e óleos - 2a 3 porções

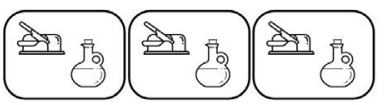

1 pão (50 g)

5 bolachas $(35 \mathrm{~g})$

1 batata e meia (125 g)

5 colheres de sopa de cereais de pequeno almoço $(35 \mathrm{~g})$ 2 (cru) / 4 (cozido) colheres de sopa de arroz/massa (35 g/110g)

1 (cozinhado) / 2 (cru) chávenas (140 g/180 g)

1 ovo $(50 \mathrm{~g})$

$30 \mathrm{~g}$ de carne ou pescado

1 peça de fruta média ( $160 \mathrm{~g}$ )

1 copo de leite $(250 \mathrm{ml})$

1 iogurte liquido / 2 iogurtes sólidos ( $250 \mathrm{~g}$ )

2 fatias de queijo ( $40 \mathrm{~g}$ )

1 (cru)/3 (cozido) colheres de sopa de leguminosas secas ( $25 \mathrm{~g} / 80 \mathrm{~g}$ ) 3 colheres de sopa de leguminosas frescas (80 $\mathrm{g}$ )

1 colher de sopa de óleo / azeite (10 g) 1 colher de chá de manteiga $(15 \mathrm{~g})$

* - valores médios para um adulto, adaptado de Roda dos Alimentos, disponivel em https://alimentacaosaudavel.dgs.pt/roda-dos-alimentos/ 
Exemplo de um registo diário para uma semana

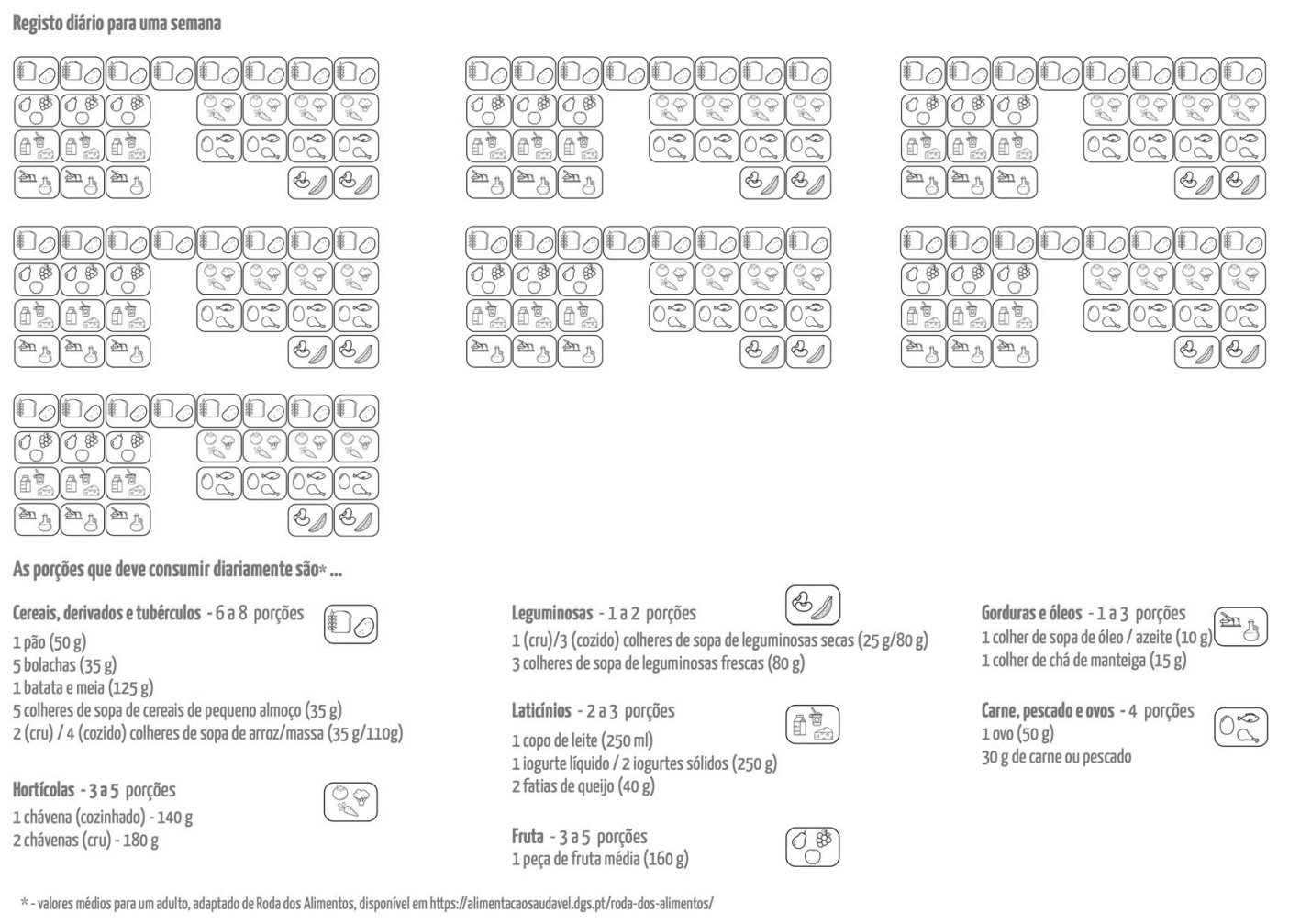

\section{Figura 9}

Detalhe do exemplo do preenchimento do registo diário/semanal

Exemplo

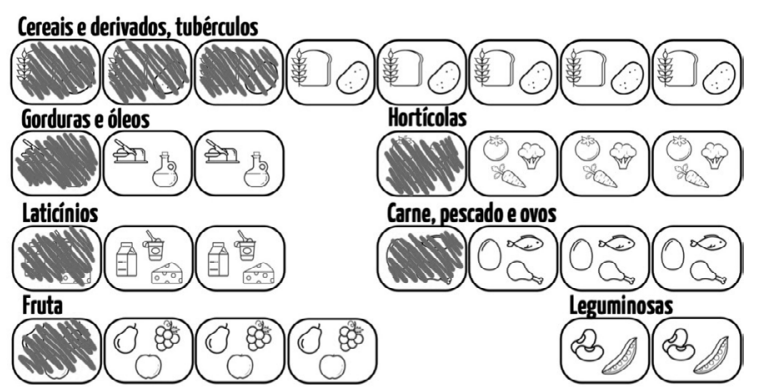

\section{Pequeno almoço}

1 iogurte líquido

1 pão com manteiga

\section{Meio da manhã \\ 1 maçã, 2 bolachas}

\section{Almoço - salada colorida com hortícolas e ovo \\ ( 6 colheres de sopa de massa, 1 ovo, $150 \mathrm{~g}$ de legumes, 1 c. sopa de azeite)}

Acresce ainda que esta disrupção pode induzir o consumidor de que é lícito ou desejável substituir os alimentos por substitutos alimentares, fontes de nutrientes $(11,13)$.

A abordagem proposta apresenta algumas limitações relacionadas com o instrumento em que se suporta - a Roda dos Alimentos, uma vez que esta não contempla, nos seus grupos, os frutos oleaginosos, alimentos que devem fazer parte de uma alimentação equilibrada. Mesmo, a Roda da Alimentação Mediterrânica, apesar de os referenciar, não define a respetiva porção, ficando inerente a ligação desta à sua versão anterior. Este facto constitui uma oportunidade para a consolidação destes dois guias alimentares, integrando as recomendações num só documento, assim como a inclusão das porções para os frutos oleaginosos.

Os diversos exemplos apresentados, revelam a forma como esta ferramenta pode ser aplicada e consistir num auxiliar eficaz para o nutricionista em todos os contextos de atuação profissional (nutrição clínica, alimentação coletiva e restauração, nutrição comunitária e saúde pública), permitindo, de forma facilmente compreensível, transmitir a informação aos diversos interlocutores.

\section{CONCLUSÕES}

Mais importante do que somar nutrientes, há que evidenciar os alimentos ingeridos e como eles se completam na proporção relativa às recomendações alimentares.

A disponibilização desta informação nas ementas, menus, receitas, entre outros, assim como nos rótulos dos produtos alimentares permitiria facilitar a consciencialização da quantidade consumida em relação às porções diárias, assim como melhorar a perceção do que é uma porção para os vários tipos de alimentos, e de como o conjunto das diferentes refeições e produtos alimentares permitem atingir as recomendações alimentares e nutricionais.

\section{REFERÊNCIAS BIBLIOGRÁFICAS}

1. da Saúde M, editor. Retrato da Saúde 2018 [Internet]. 2018 Apr. Available from: https://www.sns.gov.pt/wp-content/uploads/2018/04/RETRATO-DA-SAUDE_2018_ compressed.pdf

2. Forouzanfar MH, Afshin A, Alexander LT, Anderson HR, Bhutta ZA, Biryukov S, et al. Global, regional, and national comparative risk assessment of 79 behavioural, environmental and occupational, and metabolic risks or clusters of risks, 1990â€"2015: 
a systematic analysis for the Global Burden of Disease Study 2015. The Lancet [Internet]. 2016 Oct 8;388(10053):1659-724. Available from: http://dx.doi.org/10.1016/ S0140-6736(16)31679-8.

3. Martínez-González M, Hershey M, Zazpe I, Trichopoulou A. Transferability of the Mediterranean Diet to Non-Mediterranean Countries. What Is and What Is Not the Mediterranean Diet. Nutrients [Internet]. 2017 Nov 1;9(11):1214-26. Available from: http://www.mdpi.com/2072-6643/9/11/1226.

4. D'Innocenzo S, Biagi C, Lanari M. Obesity and the Mediterranean Diet: A Review of Evidence of the Role and Sustainability of the Mediterranean Diet. Nutrients [Internet]. 2019 Jun 1;11(6):1306-25. Available from: https://www.mdpi.com/2072-6643/11/6/1306. 5. Hemphill L. Sustainable Food Systems for Preventative and Prescriptive Medicine. 2017 Mar 3;1-3. Available from: papers3://publication/uuid/D9785AB3-551F-4E18AF9C-46AF9E976065.

6. Berry EM. Mediterranean Diet: From a Healthy Diet to a Sustainable Dietary Pattern. Frontiers in Nutrition [Internet]. 2015 May 7;2(12A):2287-8. Available from: http://www.frontiersin.org/ Nutrition_and_Environmental_Sustainability/10.3389/fnut.2015.00015/abstract.

7. Almeida M, Oliveira A. Mediterranean and atlantic dietary patterns-an approach to key characteristics and health effects. Acta Portuguesa de Nutrição [Internet]. 2017 Jan 1;11:22-8. Available from: http://actaportuguesadenutricao.pt/edicoes/edicao-no11/. 8. Chen X, Zhang Z, Yang H, Qiu P, Wang H, Wang F, et al. Consumption of ultraprocessed foods and health outcomes: a systematic review of epidemiological studies. Nutrition Journal [Internet]. 2020 Aug 20;19(1):86. Available from: https://nutritionj. biomedcentral.com/articles/10.1186/s12937-020-00604-1.

9. James SL, Abate D, Abate KH, Abay SM, Abbafati C, Abbasi N, et al. Global, regional, and national incidence, prevalence, and years lived with disability for 354 diseases and injuries for 195 countries and territories, 1990âE“2017: a systematic analysis for the Global Burden of Disease Study 2017. The Lancet [Internet]. 2018 Nov 10;392(10159):1789-858. Available from: http://dx.doi.org/10.1016/S01406736(18)32279-7.

10. Nielsen. What's in our food and on our mind [Internet]. Ingredient and dining-out trends around the world. 2016 Aug. Available from: https://nutrimento.pt/activeapp/wp-content/ uploads/2016/09/global-ingredient-and-out-of-home-dining-trends-aug-2016.pdf.

11. Mozaffarian D, Rosenberg I, Uauy R. History of modern nutrition science-implications for current research, dietary guidelines, and food policy. BMJ [Internet]. 2018 Jun 13;361:k2392. Available from: https://www.bmj.com/lookup/doi/10.1136/bmj.k2392. 12. Porto-Arias JJ, Lorenzo T, Lamas A, Regal P, Cardelle-Cobas A, Cepeda A. Food patterns and nutritional assessment in Galician university students. Journal of Physiology and Biochemistry [Internet]. 2017 Aug 3;74(1):1-8. Available from: http:// link.springer.com/10.1007/s13105-017-0582-0.

13. Mozaffarian D, Ludwig DS. Dietary guidelines in the 21 st century--a time for food. JAMA [Internet]. 2010 Aug 11;304(6):681-2. Available from: http://jama.jamanetwork. com/article.aspx?doi=10.1001/jama.2010.1116.

14. Colby SE, Johnson L, Scheett A, Hoverson B. Nutrition marketing on food labels. Journal of Nutrition Education and Behavior [Internet]. 2010 Mar 1;42(2):92-8. Available from: https://linkinghub.elsevier.com/retrieve/pii/S1499404608008476.

15. Lando AM, Labiner-Wolfe J. Helping consumers make more healthful food choices: consumer views on modifying food labels and providing point-of-purchase nutrition information at quick-service restaurants. Journal of Nutrition Education and Behavior [Internet]. 2007 May 1;39(3):157-63. Available from: http://eutils.ncbi.nlm.nih.gov/ entrez/eutils/elink.fcgi?dbfrom=pubmed\&id=17493566\&retmode=ref\&cmd=prlinks. 16. Farrukh. Metabolic Impact of the Amount and Type of Dietary Carbohydrates on the Risk of Obesity and Diabetes. 2012 Jan 9;1-14. Available from: https://www. academia.edu/22040485/Metabolic_Impact_of_the_Amount_and_Type_of_Dietary Carbohydrates_on_the_Risk_of_Obesity_and_Diabetes?auto=download.

17. Khan TA, Sievenpiper JL. Controversies about sugars: results from systematic reviews and meta-analyses on obesity, cardiometabolic disease and diabetes. European Journal of Nutrition [Internet]. 2016 Nov 30;55(s2):25-43. Available from: papers3:// publication/doi/10.1007/s00394-016-1345-3.

18. Gardner CD, Trepanowski JF, del Gobbo LC, Hauser ME, Rigdon J, Ioannidis JPA, et al. Effect of Low-Fat vs Low-Carbohydrate Diet on 12-Month Weight Loss in
Overweight Adults and the Association With Genotype Pattern or Insulin Secretion. JAMA [Internet]. 2018 Feb 20;319(7):613-67. Available from: http://jama.jamanetwork. com/article.aspx?doi=10.1001/jama.2018.0245.

19. Reeves S, Wake Y, Zick A. Nutrition labeling and portion size information on children's menus in fast-food and table-service chain restaurants in London, UK. Journal of Nutrition Education and Behavior [Internet]. 2011 Nov 1;43(6):543-7. Available from: https://linkinghub.elsevier.com/retrieve/pii/S1499404610006020.

20. Franchini B, Rodrigues S. A nova Roda dos Alimentos [Internet]. 2015. Available from: http://www.fao.org/3/a-ax433o.pdf.

21. Frobisher C, Maxweel SM. The estimation of food portion sizes: a comparison between using descriptions of portion sizes and a photographic food atlas by children and adults. Journal of Human Nutrition and Dietetics [Internet]. 2003 May 9;16:181-8. Available from: https://www.readcube.com/.

22. Biltoft-Jensen A, Holmgaard Nielsen T, Hess Ygil K, Christensen T, Fagt S. Accuracy of food photographs for quantifying food servings in a lunch meal setting among Danish children and adults. Journal of Human Nutrition and Dietetics [Internet]. 2017 Jun 27;31(1):131-40. Available from: http://doi.wiley.com/10.1111/jhn.12490.

23. Nutrimento, editor. A Roda da Alimentação Mediterrânica [Internet]. 2020. p. 1. Available from: https://nutrimento.pt/activeapp/wp-content/uploads/2016/06/Cartaz-A3-13_med.pdf. 24. (EFSA) EFSA. Dietary Reference Values for nutrients Summary report. EFSA Supporting Publications [Internet]. 2019 Sep 20;14(12):1133-98. Available from: http:// doi.wiley.com/10.2903/sp.efsa.2017.e15121.

25. McCrickerd K, Forde CG. Sensory influences on food intake control: moving beyond palatability. Obesity reviews : an official journal of the International Association for the Study of Obesity [Internet]. 2016 Jan 1;17(1):18-29. Available from: https://onlinelibrary. wiley.com/doi/full/10.1111/obr.12340.

26. Fiszman S, Tarrega A. Expectations of food satiation and satiety reviewed with special focus on food properties. Food \& Function [Internet]. 2017 Aug 1;8(8):2686-97. Available from: http://eutils.ncbi.nlm.nih.gov/entrez/eutils/elink. fcgi?dbfrom=pubmed\&id=28686245\&retmode=ref\&cmd=prlinks.

27. Chambers L. Food texture and the satiety cascade. Nutrition bulletin [Internet]. 2016 Aug 14;41(3):277-82. Available from: http://doi.wiley.com/10.1111/nbu.12221. 28. Graça P, Silva AJ. NUTR-HIA Improving nutrition labelling in Portugal Health Impact Assessment [Internet]. de Saúde DG, editor. 2019 Dec. Available from: https://www. dgs.pt/documentos-e-publicacoes/nutr-hia-improving-nutrition-labelling-in-portugalhealth-impact-assessment-final-report-pdf.aspx. 